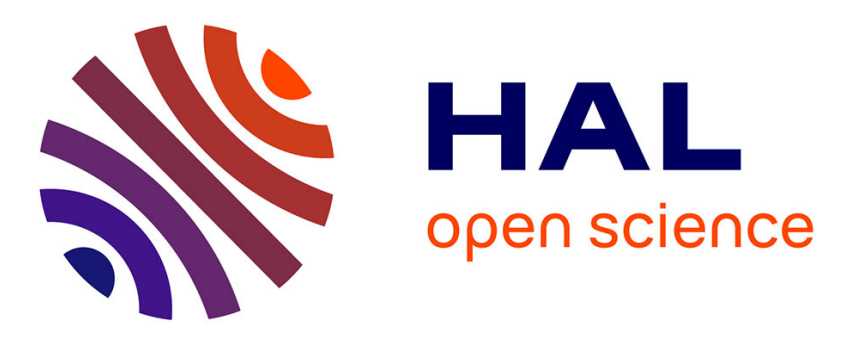

\title{
Quasi-Optical Excitation of a Circularly-Polarized Metasurface Antenna at K-band
}

Jorge Ruiz-Garcia, M. Faenzi, A. Mahmoud, Mauro Ettorre, Patrick Potier, Philippe Pouliguen, Ronan Sauleau, D. Gonzalez-Ovejero

\section{- To cite this version:}

Jorge Ruiz-Garcia, M. Faenzi, A. Mahmoud, Mauro Ettorre, Patrick Potier, et al.. Quasi-Optical Excitation of a Circularly-Polarized Metasurface Antenna at K-band. 14th European Conference on Antennas and Propagation, EuCAP 2020, Mar 2020, Copenhagen, Denmark. pp.9135303, 10.23919/EuCAP48036.2020.9135303 . hal-02931846

\section{HAL Id: hal-02931846 \\ https://hal.science/hal-02931846}

Submitted on 1 Dec 2020

HAL is a multi-disciplinary open access archive for the deposit and dissemination of scientific research documents, whether they are published or not. The documents may come from teaching and research institutions in France or abroad, or from public or private research centers.
L'archive ouverte pluridisciplinaire HAL, est destinée au dépôt et à la diffusion de documents scientifiques de niveau recherche, publiés ou non, émanant des établissements d'enseignement et de recherche français ou étrangers, des laboratoires publics ou privés. 


\title{
Quasi-Optical Excitation of a Circularly-Polarized Metasurface Antenna at K-band
}

\author{
Jorge Ruiz-García ${ }^{1}$, Marco Faenzi ${ }^{1}$, Adham Mahmoud ${ }^{1}$, Mauro Ettorre ${ }^{1}$, Patrick Potier ${ }^{2}$, Philippe Pouliguen ${ }^{3}$, \\ Ronan Sauleau ${ }^{1}$, and David González-Ovejero ${ }^{1}$. \\ ${ }^{1}$ Univ. Rennes, CNRS, IETR - UMR 6164, F-35000, Rennes, France, jorge.ruiz@univ-rennes1.fr \\ ${ }^{2}$ Direction Générale de l'Armement, 35174 Bruz, France \\ ${ }^{3}$ Direction Générale de l'Armement, Agence de l'innovation de défense, 75509 Paris, France
}

\begin{abstract}
This paper presents a new concept of modulated metasurface (MTS) antenna for satellite communications. As opposed to using cylindrical surface waves (SW) to excite circular apertures, we employ a quasi-optical beamformer to launch a plane SW. This architecture enables an efficient illumination of rectangular apertures. In addition, the use of anisotropic MTS elements allows us to obtain circularly-polarized beams with excellent characteristics in terms of cross-polarization discrimination. We present the design process of a prototype at K-band and the obtained simulation results, which prove the suitability of this antenna for satellite data links.
\end{abstract}

Index Terms-Metasurface antennas, leaky waves, circular polarization, satellite communications, CubeSats.

\section{INTRODUCTION}

Satellite communications usually require high-gain antennas with circular polarization and beam steering capabilities. In the case of moving platforms (e.g. drones and spacecrafts), these features are often obtained by employing heavy and bulky reflector antennas [1] or complex phased arrays [2]. Modulated metasurface (MTS) antennas offer an interesting alternative for some applications, since they are able to comply with the high gains required by satellite communications, while keeping a light weight and a low profile [3].

MTSs typically consist of arrangements of sub-wavelength patches on a grounded dielectric slab [4]-[7] or metallic pins grown on a metal base-plate [8]. These elements are distributed on a regular grid and their size is generally smaller than $\lambda_{0} / 6$, where $\lambda_{0}$ is the free-space wavelength. Provided that the aperture is finely discretized at a given frequency, a surface wave (SW) supported by the MTS layer will see the textured surface as a continuous impedance boundary condition (IBC). By properly modulating this equivalent IBC, one can transform the bounded SW into a leaky wave (LW), obtaining a directive radiation effect [9]. Furthermore, the pointing angle of the radiated beam can be controlled by tuning the parameters of the modulation, as explained in [6], [9].

In circular apertures, the SW launcher is usually placed at the center and utilizes a coaxial pin, so that the intrinsic cylindrical symmetry of this configuration helps to illuminate uniformly the circular area [3]-[5]. However, this configuration is not suitable when dealing with rectangular apertures, since the cylindrical wavefront is not adapted to the aperture geometry anymore. For rectangular shapes, a plane SW is needed and the feeder must be adapted. A lens can be employed to collimate a cylindrical SW [10], but an additional area is necessary to place the beamformer. A more compact possibility is the use of a pillbox quasi-optical system [11]. It consists of two substrate layers that are vertically stacked. A horn in the bottom layer is used to launch a wave that impinges on a reflector and is finally transformed into a plane SW in the top layer. Then, this plane SW can be used to illuminate a modulated MTS set on the upper layer, thus obtaining a compact MTS antenna.

The high degree of control of the aperture fields provided by modulated MTSs allows one, among other possibilities, to achieve the desired polarization for the radiated wave. In the case of satellite communications, circular polarization is commonly demanded, and this is also affordable by MTS antennas. Circular apertures have been typically employed for this purpose [3]-[5], [7], [8], [12], since the circular geometry eases the generation of circular polarization. For some specific platforms, such as CubeSats [13], [14], rectangular antennas could be more appropriate in terms of form factor agreement. Although linear LW antennas with circular polarization have been proposed [15], high-gain prototypes (directivity larger than $25 \mathrm{~dB}$ ) are not so commonly found in literature.
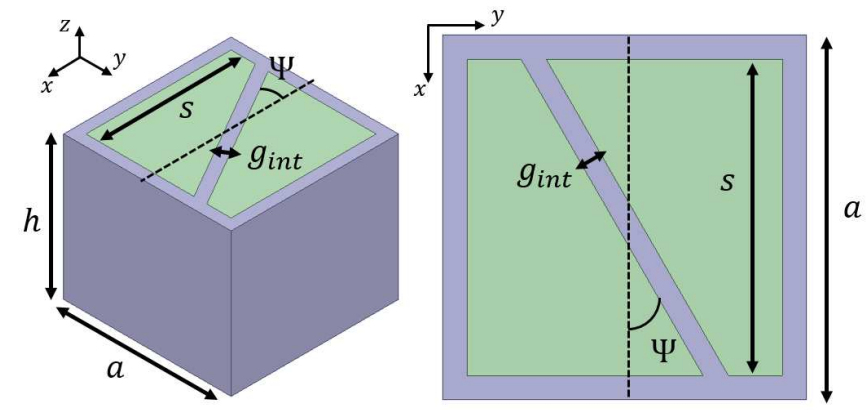

Fig. 1. Unit-cell used to implement the MTS. The fixed parameters are $h=$ $1.28 \mathrm{~mm}$ and $a=1.5 \mathrm{~mm}$, the others are swept to generate the database.

In this work, we combine a modulated MTS with a pillbox beamformer to obtain a rectangular MTS antenna with righthanded circular polarization (RHCP). To this end, we use an equivalent tensor IBC that presents an anisotropic distribution. The MTS design includes the correct choice of the metallic 


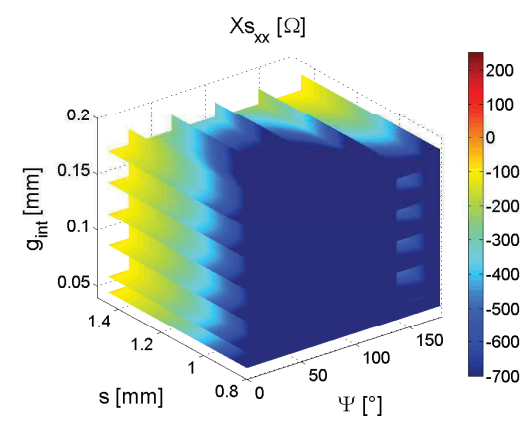

(a) $X_{x x}$

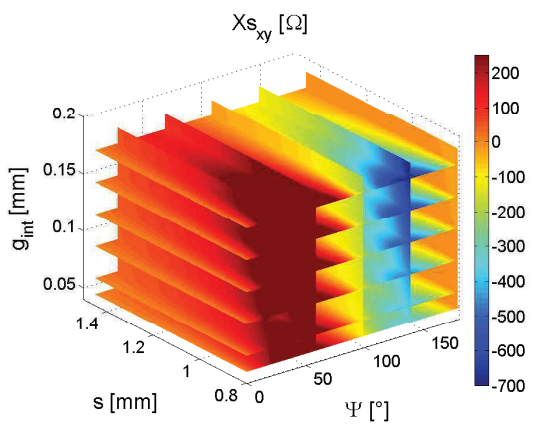

(b) $X_{x y}$

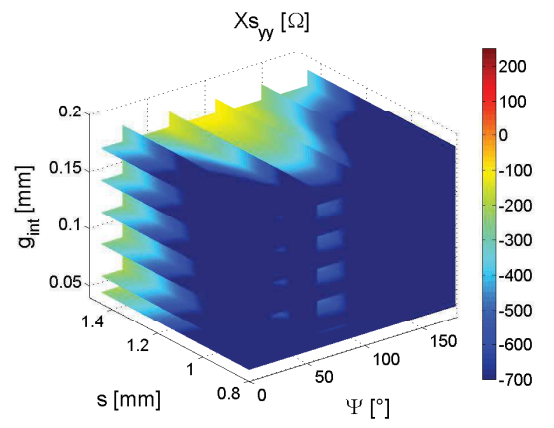

(c) $X_{y y}$

Fig. 2. 3-dimensional database relating the impedance components of (1) with the geometry of the patch in Fig. 1, where $g_{\text {int }}$ is the internal gap size, $s$ is the patch size and $\Psi$ is the gap rotation.

patch to implement the tensor IBC, as well as a suitable arrangement along a Cartesian layout. In the next section, we describe the configuration of this MTS and we show the simulation results of the final structure. Throughout the paper, we will refer to a Cartesian reference system with coordinates $(x, y, z)$ and unit vectors $(\hat{\boldsymbol{x}}, \hat{\boldsymbol{y}}, \hat{\boldsymbol{z}})$.

\section{Right-Hand Circularly-Polarized Metasurface Antenna Fed by a Pillbox BeAmformer}

Next, we present a specific design to describe the proposed architecture. In this particular case, we apply a transparent impedance [4] (or sheet transition IBC [16]) $\underline{Z}_{s}=j \boldsymbol{X}_{s}$ to introduce the dispersion effect by the grounded dielectric slab in the design procedure. We choose to direct the beam at $\theta_{0}=-5^{\circ}$ at a central frequency of $f=20.7 \mathrm{GHz}$. The employed material in both pillbox layers is Rogers RO3006 $\left(\varepsilon_{r}=6.15, \tan \delta=0.002\right)$ with thickness $d=1.28 \mathrm{~mm}$. As mentioned, we need to impose an anisotropic MTS for our proposal. Hence, we model our MTS as a reactance tensor of form

$$
\underline{\underline{\boldsymbol{X}_{s}}}=\left[\hat{\boldsymbol{x}} \hat{\boldsymbol{x}} X_{s}^{x x}+(\hat{\boldsymbol{x}} \hat{\boldsymbol{y}}+\hat{\boldsymbol{y}} \hat{\boldsymbol{x}}) X_{s}^{x y}+\hat{\boldsymbol{y}} \hat{\boldsymbol{y}} X_{s}^{y y}\right],
$$

whose tensor components are modulated along $x$ as

$$
\begin{array}{r}
X_{s}^{x x}(x)=X_{a v}(1+M \cos (2 \pi x / p)), \\
X_{s}^{x y}(x)=X_{a v}(M \sin (2 \pi x / p)), \\
X_{s}^{y y}(x)=X_{a v}(1-M \cos (2 \pi x / p)) .
\end{array}
$$

In (2), $X_{a v}$ is the average reactance, $M$ the modulation index $(0 \leq M \leq 1)$, and $p$ the period of the modulation. The form of the modulation in (1)-(2) results from the holographic product of the reference plane $\mathrm{SW}$ with the object beam pointing at $\theta_{0}$.

In order to implement (1), we use the patch shown in Fig. 1. By varying its size $s$, the internal gap size $g_{\text {int }}$ and the rotation of the gap $\Psi$, we generate the 3D database in Fig. 2, that relates the entries of the tensor IBC with the geometry of the patch. We use the method in [17] to extract the impedance value for each patch configuration. From the constructed database, we compute the geometry of the patches required to implement the space-dependent impedance in (1). The obtained MTS layout implements the ideal tensor IBC components in (2) with a relative implementation error lower than $2 \%$ for all the components of $\boldsymbol{X}_{\boldsymbol{s}}$. Then, we place this layout on the pillbox top layer to get our final structure, shown in Fig. 3. A matching section consisting of a slot array is placed after the pillbox upper parallel plate to match its impedance to that of the MTS. It is worth noting that both the SW launcher (the pillbox beamformer) and the radiating aperture (the modulated MTS) are within the same footprint. That is, we do not need any additional space to include the feeder, which makes our system very compact.

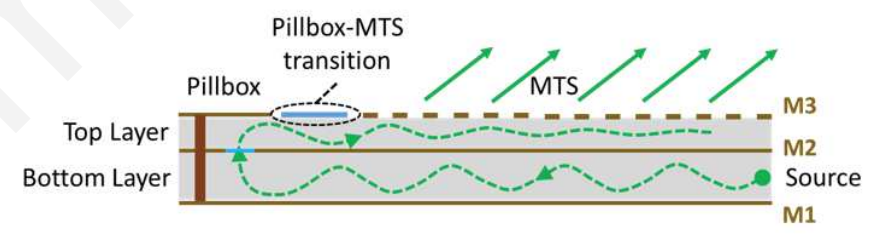

(a) Side view

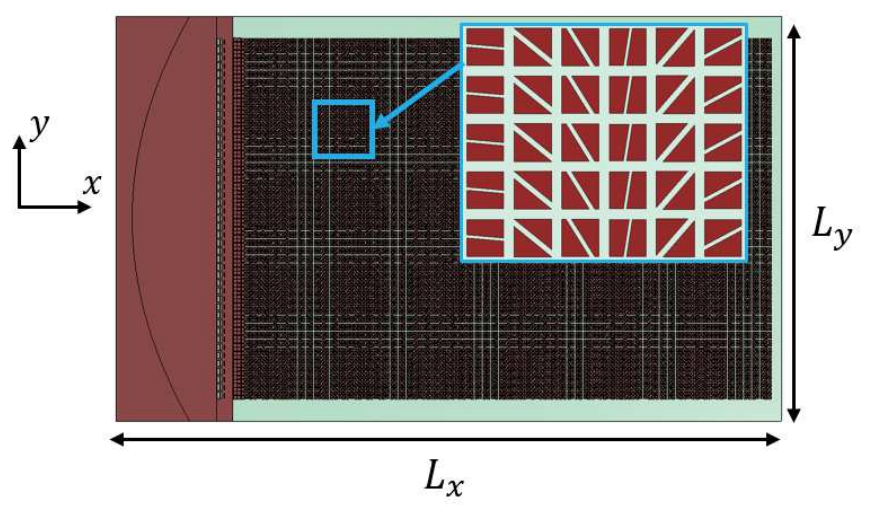

(b) Top view

Fig. 3. Final antenna configuration. The two-layer structure is represented in (a), while the modulated MTS and the pillbox top layer are shown in (b). The dimensions of the antenna are $L_{x}=17 \lambda_{0}$ and $L_{y}=10.4 \lambda_{0}$, where $\lambda_{0}$ is the free-space wavelength for $f=20.7 \mathrm{GHz}$.

Finally, the antenna is simulated with CST Microwave 


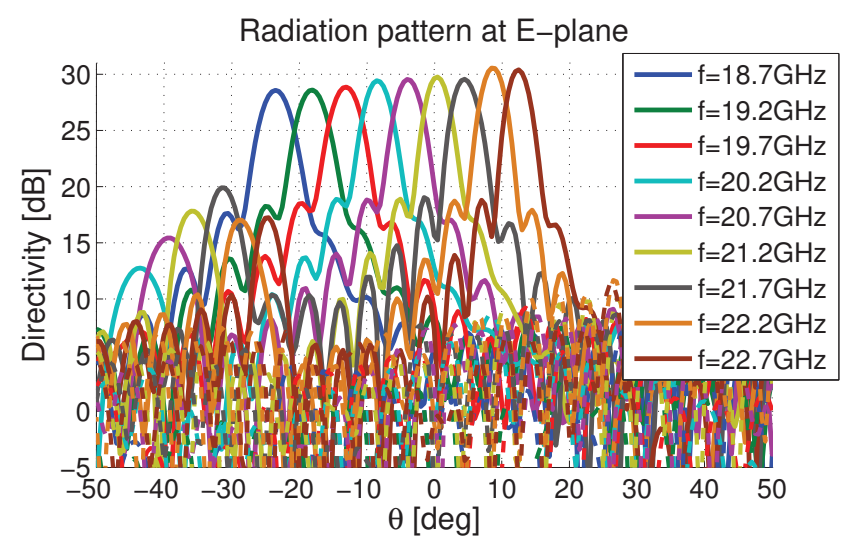

Fig. 4. Antenna radiation pattern for frequencies from $18.7 \mathrm{GHz}$ to $22.7 \mathrm{GHz}$ in steps of $0.5 \mathrm{GHz}$. Solid lines correspond to the co-polarized (RHCP) components and dash lines to the cross-polarized (LHCP) ones.

Studio. The resulting radiation patterns are shown in Fig. 4, where we include the radiated beams in the $E$-plane for different frequencies within the K-band. We can see that the directivity $D_{R H C P}$ varies between $28.6 \mathrm{~dB}$ and $30.6 \mathrm{~dB}$, so the high-gain performance is maintained for all the frequencies. This produces an aperture efficiency of $A_{e f f} \geq 50 \%$, being up to $A_{\text {eff }}=73 \%$ in the best case. The same good behaviour is observed for the cross-polarization level, which is below $-30.6 \mathrm{~dB}$ for the entire simulated band. The aperture efficiency, and then directivity, could be increased by tapering the amplitude of the aperture fields [3]-[5]. The aforementioned matching transition leads to the reflection coefficient $S_{11}$ represented in Fig. 5.

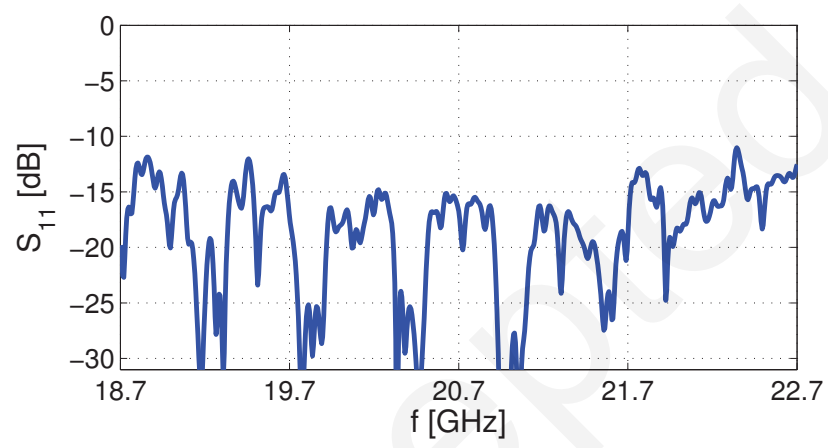

Fig. 5. Magnitude of the reflection coefficient in $\mathrm{dB}$ for the simulated antenna port.

\section{CONCLUSION}

This work presents the design of a new antenna system based on a pillbox quasi-optical beamformer and a modulated MTS. The use of a pillbox beamformer as a SW launcher produces a very compact structure, enclosing feeder and MTS in the same aperture. Our model is able to radiate a righthand circularly-polarized beam for different frequencies at the low K-band, keeping a high-gain performance $(28.6 \mathrm{~dB}<$ $\left.D_{R H C P}<30.6 \mathrm{~dB}\right)$ and a very good cross-polarization discrimination (higher than $30.6 \mathrm{~dB}$ ). Regarding the aperture effi- ciency, the presented structure offers a similar performance as MTS antennas consisting of circular apertures. Therefore, the proposed high-gain and low profile modulated MTS antenna constitutes an attractive solution for satellite communications in moving platforms. The rectangular footprint of the system makes it especially interesting for CubeSats and, in general, SmallSats applications, as it fits perfectly the form factor of these platforms.

\section{ACKNOWLEDGMENTS}

This publication has been supported by the European Union through the European Regional Development Fund (ERDF), and by the French Region of Brittany, Ministry of Higher Education and Research, Rennes Métropole and Conseil Départemental 35, through the CPER Project STIC \& Ondes. It has also been supported by the Agence d'Innovation de Défense (AID) and by Britanny Region under ARED program.

\section{REFERENCES}

[1] S. D. Targonski, "A multiband antenna for satellite communications on the move," IEEE Transactions on Antennas and Propagation, vol. 54 no. 10 , pp. 2862-2868, Oct 2006.

[2] G. Han, B. Du, W. Wu, and B. Yang, "A novel hybrid phased array antenna for satellite communication on-the-move in ku-band," IEEE Transactions on Antennas and Propagation, vol. 63, no. 4, pp. 1375 1383, April 2015.

[3] G. Minatti, M. Faenzi, E. Martini, F. Caminita, P. De Vita, D. GonzálezOvejero, M. Sabbadini, and S. Maci, "Modulated metasurface antennas for space: Synthesis, analysis and realizations," IEEE Trans. Antennas Propag., vol. 63, no. 4, pp. 1288-1300, Apr. 2015

[4] D. González-Ovejero, G. Minatti, G. Chattopadhyay, and S. Maci, "Multibeam by metasurface antennas," IEEE Trans. Antennas Propag. vol. 65, no. 6, pp. 2923-2930, Jun. 2017.

[5] M. Faenzi et al., "Metasurface antennas: New models, applications and realizations," Sci. Rep., vol. 9, p. 10178, July 2019.

[6] A. M. Patel and A. Grbic, "A printed leaky-wave antenna based on a sinusoidally-modulated reactance surface," IEEE Trans. Antennas Propag., vol. 59, no. 6, pp. 2087-2096, Jun. 2011.

[7] B. Fong, J. Colburn, J. Ottusch, J. Visher, and D. Sievenpiper, "Scalar and tensor holographic artificial impedance surfaces," IEEE Trans. Antennas Propag., vol. 58, no. 10, pp. 3212-3221, Oct. 2010.

[8] D. González-Ovejero, N. Chahat, R. Sauleau, G. Chattopadhyay, S. Maci, and M. Ettorre, "Additive manufactured metal-only modulated metasurface antennas," IEEE Transactions on Antennas and Propagation, vol. 66, no. 11, pp. 6106-6114, Nov 2018.

[9] A. Oliner and A. Hessel, "Guided waves on sinusoidally-modulated reactance surfaces," IRE Trans. Antennas Propag., vol. 7, no. 5, pp. 201-208, Dec. 1959.

[10] Y. B. Li et al., "Dual-physics manipulation of electromagnetic waves by system-level design of metasurfaces to reach extreme control of radiation beams," Adv. Mater. Technol., vol. 2, no. 1, p. 1600196, 2017.

[11] M. Ettorre, R. Sauleau, and L. Le Coq, "Multi-beam multi-layer leakywave siw pillbox antenna for millimeter-wave applications," IEEE Transactions on Antennas and Propagation, vol. 59, no. 4, pp. 1093 1100, April 2011.

[12] G. Minatti, F. Caminita, E. Martini, M. Sabbadini, and S. Maci, "Synthesis of modulated-metasurface antennas with amplitude, phase, and polarization control," IEEE Trans. Antennas Propag., vol. 64, no. 9, pp. 3907-3919, Sept 2016.

[13] N. Chahat, R. E. Hodges, J. Sauder, M. Thomson, E. Peral, and Y. Rahmat-Samii, "CubeSat deployable Ka-band mesh reflector antenna development for Earth science missions," IEEE Trans. Antennas Propag., vol. 64, no. 6, pp. 2083-2093, Jun. 2016.

[14] N. Chahat et al., "Advanced cubesat antennas for deep space and earth science missions: A review," IEEE Antennas and Propagation Magazine, vol. 61 , no. 5 , pp. $37-46$, Oct 2019. 
[15] S. Otto, Z. Chen, A. Al-Bassam, A. Rennings, K. Solbach, and C. Caloz, "Circular polarization of periodic leaky-wave antennas with axial asymmetry: Theoretical proof and experimental demonstration," IEEE Transactions on Antennas and Propagation, vol. 62, no. 4, pp. 1817-1829, April 2014.

[16] A. M. Patel and A. Grbic, "Effective surface impedance of a printed- circuit tensor impedance surface (PCTIS)," IEEE Trans. Microw. Theory Tech., vol. 61, no. 4, pp. 1403-1413, April 2013.

[17] A. M. Patel and A. Grbic, "Modeling and analysis of printed-circuit tensor impedance surfaces," IEEE Transactions on Antennas and Propagation, vol. 61, no. 1, pp. 211-220, Jan 2013. 